\title{
Commentary
}

\section{An emerging toolkit for targeted cancer therapies}

\author{
Gordon B. Mills \\ The University of Texas MD Anderson Cancer Center, Houston, Texas 77030, USA
}

\begin{abstract}
The investment in the "War on Cancer" initiated by the National Cancer Act of 1971 is paying off in terms of improved patient outcomes, with more than $65 \%$ of all cancer patients now surviving more than five years in developed countries (Howlader et al. 2011). The number of cancer survivors now exceeds 11 million in the United States alone, with one in 30 adults being a cancer survivor (Howlader et al. 2011). Due to the intensive research programs focused on cancer, our understanding of the processes leading to the initiation and progression of cancer has improved markedly, and this, in turn, has fueled the transition to rational approaches to therapy by targeting the effects of the underlying genomic events driving the pathophysiology of cancer rather than previously relatively non-specific and more toxic chemotherapy approaches. This progress has been hard won in terms of the number of patients who have contributed by participating in clinical trials and certainly has been much slower than hoped for. However, a rapidly emerging toolkit is creating a "perfect storm" of deep mechanistic understanding, relevant model systems, an array of targeted therapeutics, and technological advances allowing the characterization of the patient and tumor genome that are the topics of this special issue of Genome Research and that should herald an unprecedented rate of improvement in patient outcomes.
\end{abstract}

\section{An emerging technological toolkit}

A technological revolution is driving the expectation that we are on the verge of a transformation of approaches to cancer management with an attendant improvement in patient outcomes. While approaches able to characterize the proteome and metabalome are beginning to emerge, next-generation sequencing technologies able to comprehensively evaluate DNA and RNA changes in tumors have altered the way in which we characterize tumors and manage patients (Harris and McCormick 2010; Macconaill and Garraway 2010; Chin et al. 2011; Roychowdhury et al. 2011). Next-generation sequencing now makes it possible to assess DNA copy number, rearrangements, loss of heterozygosity, allele-specific amplification, methylation, transcription, aberrant splicing, and RNA editing with a single high-throughput, costeffective platform. New approaches and applications described in this issue are extending the utility of next-generation sequencing (Thompson et al. 2012). The concepts that the "actionable" events in a tumor can already be characterized for less than a thousand dollars and that the " $\$ 1000$ genome" is nearing reality would have been considered heretical only a generation ago. These new sequencing technologies are providing, for the first time, the potential to characterize both the patient and the tumor genome to aid in therapy planning and to deliver on the potential of personalized molecular medicine. This special issue on Cancer Genomics brings together a series of articles that highlight the issues

Article and publication date are at http://www.genome.org/cgi/doi/ 10.1101/ gr.136044.111. involved in the implementation of next-generation sequencing technologies for an improved understanding of the mechanisms leading to the development of cancer, the spectrum of mutations and chromosomal aberrations in tumors, the role of the epigenome, approaches to determine which mutations and copy number changes are drivers and thus, actionable, and improved methods to identify cancer-relevant mutations and copy number changes.

However, despite the incredible enthusiasm engendered by the promise of personalized cancer therapy, many challenges remain to be overcome before targeted therapies can become the standard of practice benefiting all cancer patients.

The implementation of personalized cancer therapy will require an improved link between the ability to characterize the genome of the patient and the changes that occur in the patient's tumor to identify biomarkers that will predict which patients will respond to specific targeted therapeutics (Brooks 2012). Improvements in drug development, such as that of therapeutic antibodies and small molecule inhibitors, and emerging approaches to interrupt protein-protein and protein-DNA interactions, such as stapled peptides, and siRNA approaches to silence gene expression may soon allow us to drug the "undruggable" and are creating a new drug toolkit (Oltersdorf et al. 2005; Verdine and Walensky 2007; Mangala et al. 2009). Furthermore, the concept of "cotherapy" trials in which the same drugs are concurrently explored in patients and relevant animal models is greatly increasing our ability to validate biomarkers able to identify cancer patients likely to benefit and at the same time to characterize mechanisms underlying sensitivity and resistance (Olive and Tuveson 2006; Liu et al. 2011). These studies have been particularly useful in dissecting resistance mechanisms that can then lead to rational combinatorial therapy.

The Cancer Genome Atlas (TCGA, http://cancergenome.nih. gov) and the International Cancer Genome Consortium (ICGC, http://www.icgc.org) as well as many other smaller programs are generating a parts list of the genomic alterations, changes in transcriptional profiles, and to a lesser degree proteomics patterns that occur across a wide variety of cancer lineages. Indeed, it is no longer appropriate to implement clinical trials and manage cancer patients solely based on tissue of origin. Rather, an integration of the underlying genomic events driving tumor initiation and progression with the intrinsic gene expression patterns and context of the tissue of origin is driving a greater understanding of tumor pathophysiology and how this should alter treatment approaches. While several new candidate cancer genes have been discovered by these efforts, perhaps the most important information arising from these efforts relates to the frequency and types of aberrations driving particular tumor lineages and how these can be targeted for improved patient management. For example, while endometrioid endometrial cancer appears to be primarily a disease of point mutations and indels, high-grade serous ovarian cancer appears to be primarily a disease of copy number change driven by almost universal defects in TP53 and frequent aberrations in homologous recombination, primarily through germline and somatic defects 
in BRCA1 and BRCA2 (http://cancergenome.nih.gov, Cancer Genome Atlas Research Network 2011). The revelation that ductal breast cancers, which were thought to be indistinguishable based on light microscopy, were indeed different diseases requiring different approaches to therapy has been promulgated across multiple tumor lineages. Indeed, cross tumor comparison is demonstrating that different tumor lineages such as basal breast cancers, high-grade serous ovarian cancers, and serous endometrial cancers have more in common across tumor lineages than within tumor lineages with their counterparts of luminal breast cancer, low-grade ovarian cancer, and endometrioid endometrial cancer, respectively (http://cancergenome.nih.gov). Whether common genomic events across cancer lineages will translate into response to similar therapies remains to be fully tested but, if validated, could hasten the implementation of effective therapy approaches. Furthermore, this information could lead to new trial designs in which underlying genomic changes rather than tissues are the most critical step in patient selection. Large-scale sequencing efforts have demonstrated remarkable heterogeneity both within and between tumors and between primary tumors and metastases (Gonzalez-Angulo et al. 2011; Jensen et al. 2011; Turajlic et al. 2012). Furthermore, they have begun to elucidate the mechanisms underlying the genomic instability in tumors (Castellarin et al. 2012; Guenthoer et al. 2012; Hinoue et al. 2012; Kostic et al. 2012; Wang et al. 2012; Xiong et al. 2012; Xu et al. 2012). A concerted community effort will be necessary to translate the data generated by the TCGA and the ICGC and other sequencing efforts into the knowledge and wisdom that will be required to improve patient outcomes.

\section{Learning from our successes}

\section{Linking biomarkers to targeted therapy}

A number of targeted therapies have been implemented into standard of care (Table 1). Remarkably, in almost all cases, biomarkers able to identify patients most likely to respond have been critical to the successful implementation of targeted therapies. In most cases, the mechanism of response appears to be due to "oncogene addiction," a term coined by I.B. Weinstein to indicate the dependence of the tumor on continued activity of a single cancer

Table 1. Most effective targeted therapies are linked to markers of efficacy

\begin{tabular}{|c|c|c|c|}
\hline Imatinib mesylate & CML & $B C R-A B L$ translocation & Oncogene addiction (1982) \\
\hline Imatinib mesylate & GIST & KIT mutation & Oncogene addiction (1999) \\
\hline Sunitinib & $\begin{array}{l}\text { Dermatofibrosarcoma } \\
\text { protuberans }\end{array}$ & & \\
\hline Nilotinib & $\begin{array}{l}\text { Hypereosinophylic } \\
\text { syndrome }\end{array}$ & PDGFRA mutation & \\
\hline Dasatinib & Melanoma & & \\
\hline Trastuzumab & Breast cancer & HER2 amplification & Oncogene addiction (1985) \\
\hline Pertuzumab & & & \\
\hline Lapatinib & & & \\
\hline Gefitinib, erlotanib & Lung cancer & EGFR mutation & Oncogene addiction (2004) \\
\hline Cetuxumab & Bowel cancer & & \\
\hline $\begin{array}{l}\text { PKC412, SU11248, } \\
\text { CMT53518 }\end{array}$ & $\mathrm{AML}, \mathrm{ALL}$ & $\begin{array}{l}\text { FLT3 mutation, } \\
\text { tandem duplication }\end{array}$ & Oncogene addiction (1996) \\
\hline PARP inhibitors & $\begin{array}{l}\text { Breast cancer } \\
\text { Ovarian cancer }\end{array}$ & $B R C A 1 / 2$ mutation & Synthetic lethality (2005) \\
\hline PLX4032 & Melanoma & $B R A F(8 \mathrm{yr})$ & Oncogene addiction (2002) \\
\hline Crizotinib & Lung cancer & $E M L 4-A L K(4 \mathrm{yr})$ & Oncogene addiction (2007) \\
\hline Tamoxifen, Als & Breast cancer & ER expression & Lineage (1800s) \\
\hline
\end{tabular}

gene and in most cases an oncogene (Weinstein 2002). This concept has now been extended to the term "driver" representing a genomic event that drives tumor initiation or progression as compared with "passengers," genomic events that occur due to the genomic instability that is inherent to many cancers (Bozic et al. 2010). This concept is critically important as we begin to use nextgeneration sequencing to select patients for targeted therapy trials in which linking effective drugs to underlying driver events will be critical to improved patient outcomes.

The major success heralded by the remarkable activity of imatinib mesylate in chronic myelogenous leukemia (CML) and in gastrointestinal stromal tumor (GIST) was exceeding informative (Druker 2008; Liegl-Atzwanger et al. 2010). However, many of the lessons of the CML story have only recently become apparent. CML is primarily driven by a single major event, the $B C R-A B L$ translocation. Indeed, the incredible responsiveness of CML may occur in part due to the diagnosis of CML at an early stage when there are a limited number of genomic aberrations, similar to what is seen in "in situ" disease such as lobular carcinoma in situ (LCIS) or ductal carcinoma in situ (DCIS) in solid tumors. Indeed, once CML enters the "blast crisis" characterized by acquisition of marked genomic instability, responses to imatinib mesylate are less frequent and generally of shorter duration. In GIST, it is clear that not all mutations in KIT are created equal. Indeed, the frequency and duration of response is markedly different with mutation in different domains of KIT (Liegl-Atzwanger et al. 2010). This concept has now been confirmed in recent studies in melanoma in which only recurrent aberrations and evidence for selection of the aberration signal a high likelihood of response (Carvajal et al. 2011). In this issue, a novel approach to determining which alleles have been selected in tumors could provide a useful approach to identify tumor aberrations that have been selected during tumor progression and are more likely to be driver aberrations (Dewal et al. 2012) and thus signal responses to targeted therapies.

The convergence of the discovery of the EML4-ALK fusion gene and the presence of crizotinib in the emerging drug toolkit may indicate a hope for rapid progress in the future (Gerber and Minna 2010). In contrast to most of the examples in Table 1 of targeted therapy where many years transpired between discovery of the abnormality and the approval of an effective targeted therapy, the time from discovery of $E M L 4-A L K$ to approval of crizotinib was under five years. With the toolkit of drugs under development in industry and academia to target specific kinases combined with off-target activity of many of these kinase inhibitors blocking additional kinases, we now have drugs that effectively inhibit almost all kinases. Because mutations, fusion genes, and changes in copy number fusions are demonstrated to alter the activity of these kinases, it may now be possible to rapidly provide proof of concept studies that a new kinase aberration is a "driver" that would lead to rapid implementation in clinical trials, and, if validated, into standard of care.

PARP inhibitors underline an important new concept. While it has been difficult to repair defects in tumor suppressors or reconstitute their functions, targeting emergent dependencies of tumor

\section{Genome Research \\ www.genome.org}


cells engendered by the defective tumor-suppressor gene is very attractive (Lord and Ashworth 2008). In the case of BRCA1/2 mutations or silencing, the resultant defects in homologous recombination render cells sensitive to the effects of PARP inhibitors by limiting the repertoire of approaches available to the tumor cell to repair DNA damage. In contrast, the "redundant" DNA repair pathways in normal cells are expected to render them resistant to the effects of PARP inhibitors. Indeed, this concept of synthetic interactions is being tested with oncogenes such as RAS and MYC that have proven difficult to target by conventional approaches (Weidle et al. 2011).

\section{Targeting patients to Phase I trials demonstrates proof} of concept and activity

Our ability to characterize the majority of aberrations in actionable cancer genes has made it possible to rapidly test potential biomarkers based on targeting the patients most likely to benefit in the trial. In Phase I Trials at MD Anderson Cancer Center, patients with aberrations in members of the phosphatidylinositol 3 kinase (PI3K) pathway were matched to Phase I trials with drugs targeting the pathway (Janku et al. 2011; Tsimberidou et al. 2011). Remarkably, 29\% of patients demonstrated marked response when the underlying genomic aberration was matched to therapy. In contrast, only $8 \%$ of patients responded when receiving the same therapies in the absence of the underlying genomic aberration, and only $6 \%$ of patients responded when their tumor was not tested for underlying genomic aberrations. This suggests that even in Phase I trials, linking underlying genomic aberrations to targeted therapies can result in improved patient responses. A deeper look at the data suggests that there is a link between lineage and response with many of the responders having breast, ovarian, or endometrial cancers and with bowel cancer patients being less likely to respond despite the presence of PI3K pathway aberrations. Furthermore, co-occurrence of PI3K pathway aberrations and KRAS mutations was associated with limited responses. Thus, the combination of markers of sensitivity (underlying genomic aberrations in the PI3K pathway), markers of resistance (comutations in RAS), and lineage combine to provide predictors of benefit (Janku et al. 2011; Tsimberidou et al. 2011). Thus a comprehensive analysis of tumors to identify genomic coevents is likely to be required to develop truly predictive biomarkers. At the MD Anderson Cancer Center, an analysis of hot-spot mutations in 33 different genes demonstrated that fewer than $50 \%$ of patients had actionable events, i.e., changes that could be used to select therapy or clinical trials. Furthermore, $\sim 7 \%$ had comutations, i.e., aberrations in more than one of the genes assessed (data not shown). Indeed, early studies suggest that this may indeed be the case. Given the relatively low frequency of coevents in tumors, data from many patients will be required to determine the consequences of comutations. However, previous studies from our and other groups have already demonstrated that the presence of specific KRAS mutations indicates limited efficacy of EGFR-targeted therapies and that PI3K pathway aberrations are associated with lack of response to specific HER2-targeted therapies (Berns et al. 2007; Harris and McCormick 2010).

\section{Pathways and networks}

In the past, community efforts have mainly focused on mechanistic studies of individual genomic events in patient tumors such as mutations, rearrangements, or regions of genomic copy number changes. However, it is clear that tumor behavior is driven by coordinate targeting of pathways and networks. Emerging approaches to assemble changes in copy number, mutations, rearrangements, epigenetic changes, and translational profiling detected on different technological platforms, between patients, and within tumors from individual patients into pathways and networks have greatly improved our understanding of mechanisms driving tumor behavior (http://cancergenome.nih.gov; http://www.icgc.com). However, the ability to integrate and interpret data across platforms has lagged behind the ability to generate data, particularly with next-generation sequencing approaches. Two different articles in this issue provide new approaches to this challenge (Ciriello et al. 2012; Xiong et al. 2012). For example, an analysis of ovarian cancer by the TCGA demonstrated that in addition to the $12 \%-13 \%$ of patients with germline mutations in BRCA1/2, approximately half as many have somatic mutations in BRCA1/2 (Cancer Genome Atlas Research Network 2011). When mutational data are combined with assessment of changes in methylation and expression of $B R C A 1 / 2, \sim 30 \%$ of patients would be expected to demonstrate defective homologous recombination. Including mutations, copy number changes, or methylation of additional genes implicated in homologous recombination would indicate that up to half of all ovarian cancer patients have a demonstrable aberration in the BRCA1/2 pathway. Thus, considering the pathway rather than individual events or nodes in the pathway as an indicator of therapeutic liability would greatly increase the number of patients who have the potential to be entered into clinical trials and who may benefit from PARP inhibitor therapy designed to be effective in patients with defects in homologous repair.

An integrated analysis of genomic and transcriptional changes in glioma indicates that, while an aberration in any single member of the PI3K pathway is a relatively uncommon event, considering the PI3K pathway in its entirety as an indicator of potential sensitivity to PI3K pathway targeted therapy suggests that more than $80 \%$ of patients have at least one aberration and could benefit from pathway-targeted therapy (Cancer Genome Atlas Research Network 2008). Indeed, several articles in this issue focus on methods of identifying pathways targeted both across and within patients.

\section{Let the patient teach us what is important}

A small number of patients in early clinical trials, usually between $5 \%$ and $10 \%$, demonstrate remarkable responses including decreased tumor size by RECIST (response evaluation criteria in solid tumors). These patients have the potential to teach us important lessons. However, in the past we lacked adequate tools to learn from these patients. Perhaps the most cogent example was the demonstration, based on fewer than 10 patients, that major responders to EGFR-targeted therapy had mutations in the EGFR receptor gene that engendered oncogene addiction and sensitivity to the targeted therapies (Paez et al. 2004). A similar example from the MD Anderson Cancer Center demonstrated that a single patient in a melanoma trial responded to sorafinib, which was being given to target RAF1 mutations (Quintás-Cardama et al. 2008). However, it turned out that this patient's tumor did not have aberrations in RAF1 but rather had a pathogenic KIT mutation. Indeed, this and other patient, cell line, and animal studies have led to the implementation of molecular testing and KIT-targeted therapy for the subset of melanomas with mutations in KIT as standard of therapy at the MD Anderson Cancer Center. As noted 
above, however, only patients with evidence for selection of KIT mutations, including the presence of hot-spot mutations or selective allele amplification, appear to benefit from KIT-targeted therapy (Carvajal et al. 2011). This study suggests that rare mutations in the KIT gene in melanoma represent passenger mutations that occurred due to underlying genomic instability rather than being drivers of tumor behavior. Novel approaches to identify which alleles are selected during tumor progression presented in this issue may improve our ability to identify driver mutations that represent therapeutic liabilities.

\section{Challenges to clinical implementation of molecular testing in patient care}

Despite the many exciting examples of the efficacy of linking genomic aberrations to targeted therapy (Table 1), many hurdles remain to be overcome before next-generation sequencing analysis of candidate genes, whole exomes, or whole genomes can be implemented into standard of care. Several groups, including our own, have established programs designed to identify the challenges and implement approaches to overcome these challenges (Wagle et al. 2011). It is important to emphasize once again that only a portion of patients $(<50 \%)$ has potentially actionable aberrations on hot-spot mutation analysis. Whether full-length sequencing of greater numbers of candidate cancer-related genes, whole exomes, or whole genomes will alleviate this concern remains to be tested. Preliminary full-length sequencing data from a limited number of candidate genes in patient samples suggest that a greater portion of patients will have actionable mutations (Roychowdhury et al. 2011; Wagle et al. 2011) when additional genes are assessed in their entirety. Furthermore, even for patients whose tumors have a biomarker of likelihood of response to therapy (Table 1), only a portion of the patients respond, and, unfortunately in most cases, the responses are short. Thus, it will be critical to identify mechanisms of resistance including preexisting, adaptive, and acquired resistance so that rational combinatorial therapies can be developed and implemented. Indeed, it is unlikely that major progress will be made in cures of solid tumors until approaches to efficiently identify mechanisms of sensitivity and resistance and target these aberrations early in patient management are established.

\section{Accuracy of next-generation testing}

Implementation of molecular testing into patient care requires that all tests be performed in a Clinical Laboratory Improvement Amendments (CLIA)-certified laboratory with a high degree of quality control and an understanding of the accuracy of the results. Next-generation sequencing as currently implemented is errorprone with significant challenges with both false-positive and false-negative calls. Increasing the depth of sequencing can alleviate this; however, this may not be sufficient (Wagle et al. 2011). Mutation calling is more problematic in cancer diagnostics than in analysis of germline sequence due to the presence of stromal cells, infiltrating lymphocytes, and the potential presence of multiple tumor clones that alter the frequency of a particular genomic change and, in particular, mutations. As the depth of sequencing is increased, both the time and cost of obtaining data are greatly increased. Indeed, while conceptually, whole-genome sequencing offers the greatest opportunity to obtain information that could alter patient management, if deep sequencing is required, then the cost of sequencing, storage of data, and analysis of data may become prohibitive. Furthermore, because only a few aberrations are currently actionable, much of the information acquired from the whole-genome sequence will not be informative for patient care (Wagle et al. 2011). An alternative approach is to focus on deep characterization of actionable events where the significance of aberrations tested is more readily ascertained. This provides the opportunity to decrease the costs of data acquisition and analysis but limits the opportunity for discovery of important events or coevents that indicate sensitivity and resistance to targeted therapies. Currently, an efficient approach to confirm aberrations identified by deep sequencing in CLIA laboratories is needed. Indeed, Sanger and pyrosequencing as well as Sequenom MassArray or SNAPshot SNP detection, which are currently the standard approach to characterizing aberrations in patient tumors, likely lack the sensitivity to confirm aberrations identified by deep sequencing in tumors with a high degree of stromal contamination or the presence of multiple tumor clones (Wagle et al. 2011). One option is to perform detection and confirmation of aberrations in patient tumors using orthologous deep-sequencing platforms with different chemistries. It is thus urgent that improved algorithms for "calling" aberrations as well as methods for confirming aberrations identified by deep sequencing be developed to facilitate implementation of next-generation sequencing in patient management.

\section{Treating the lesion that threatens the patient}

In most cases, patient management is determined by characterization of the primary tumor. However, patient outcome is determined with a few exceptions by the pathophysiology of metastatic tumors. Given that it is technically impossible in most cases to sample all metastatic lesions and, in particular, small or dormant lesions that are not detected by current imaging technologies, it is critical to determine whether characterization of the primary tumor is able to capture the potential heterogeneity of molecular events in tumors that are distant in either space (metastases) or time (recurrences). Early studies of breast cancer suggest that there is a marked heterogeneity at least in terms of dominant clones between primary and metastatic lesions (Gonzalez-Angulo et al. 2011; Jensen et al. 2011). Indeed, heterogeneity between primary and metastatic tumors for only a limited number of markers is associated with markedly worsened outcomes (Liedtke et al. 2009). Whether this reflects a greater aggressiveness of tumors with increased genomic instability resulting in the generation of multiple subclones or failure to use effective therapy to treat the metastatic lesions remains to be determined. However, it emphasizes the need to determine whether lesions to be targeted in clinical trials are present in the metastatic or recurrent lesion(s). Studies in this issue demonstrate that, although primary and metastatic acral melanomas have similar aberrations, differences between the primary and the metastasis could influence response to therapy (Turajlic et al. 2012). Certainly, when a dominant metastatic or recurrent site is present, biopsy and deep characterization of the lesion that threatens the patient (i.e., the metastasis) should be considered both in terms of clinical trials and in terms of research to determine the impact of tumor heterogeneity. As indicated above, obtaining and characterizing a biopsy of lesions that respond or fail to respond to therapy would be critically important in increasing our understanding of both sensitivity and resistance to targeted therapy and in the subsequent development and implementation of rational combinatorial therapy aimed at overcoming resistance.

Circulating tumor cells (CTCs) and circulating free DNA (cfDNA) are released from tumors and are present in the circulation.

\section{Genome Research}


Because blood is readily accessible and it is possible that material from multiple metastatic lesions will be represented by CTCs or cfDNA in the bloodstream, CTCs or cfDNA may provide an optimal source to determine the spectrum of aberrations present in both primary and metastatic lesions present at any time during therapy. Indeed, in this issue, cfDNA has been demonstrated to be sufficiently sensitive to accurately separate patients with breast cancer from healthy controls and to reflect the aberrations present in the primary tumor (Shaw et al. 2012). Importantly, aberrations continued to be detectable in cfDNA following removal of the primary tumor, likely representing suboccult metastasis and potentially dormant lesions that could result in recurrences many years later (Shaw et al. 2012). If cfDNA or CTCs prove to accurately reflect aberrations present in tumors and in particular metastatic or recurrent tumors, this approach may obviate the number of biopsies that will need to be performed to select appropriate targeted therapies for each patient and at different times during patient care.

An alternative to dealing with heterogeneity across patients is to identify the timing of aberrations that occur in tumors (Greenman et al. 2012). Targeting early events, if druggable, that are present in all tumor cells could bypass the heterogeneity that could occur due to genomic instability and selection of clones with survival advantages during the course of tumor progression or patient therapy.

\section{Drivers and passengers}

As noted above, one of the major challenges in sequencing of patient tumors and changing patient management is distinguishing drivers from passengers and determining how to treat the spectrum of drivers present in each tumor. Many algorithms have been developed that are designed to identify driver versus passenger mutations. An even greater challenge will be in developing approaches to determine which mutations engender sensitivity to specific targeted therapeutics. Indeed, a subset of mutations in PIK3CA and PIK3R1 that encode the catalytic subunit and regulatory subunit of phosphatidylinositol 3 kinase (PI3K) has been demonstrated to engender sensitivity to therapeutics targeting the PI3K pathway (Cheung et al. 2011). However, other mutations in these genes appear to result in sensitivity to therapeutics targeting only a subset of nodes in the PI3K pathway. However, in some cases, aberrations in these proteins do not seem to be associated with sensitivity to drugs targeting the PI3K pathway either because they are passengers or alternatively as they create dependencies on different pathways by functioning as neomorphs (data not shown).

As our ability to characterize the genome of tumors from specific patients improves, the number of aberrations discovered and the challenge to determine their relevance to response to targeted therapies will increase rapidly. This demands the development of bioinformatic approaches to determine which aberrations are likely to be drivers, efficient informatic approaches to probe the literature and databases to suggest which therapies are likely to be effective, and critically experimental approaches designed to iteratively test and refine predictions from the algorithms. In this issue, a new approach called Dendrix is proposed as a method to identify potential driver mutations, driver genes, and driver pathways (Vandin et al. 2012). Combining these approaches to determine which potential drivers are early events and where there is evidence for selection could function to improve the ability to detect and target driver aberrations (Dewal et al. 2012; Greenman et al. 2012).

\section{Conclusions}

We are entering an extremely exciting time, when for the first time we have the ability to characterize the tumor and patient genome. Linking this newfound information to an emerging toolkit of targeted therapies is likely to improve patient outcomes. However, many challenges will need to be overcome before all patients will benefit from personalized cancer therapy and, in particular, benefit with durable responses and cures with limited toxicity.

\section{References}

Berns K, Horlings HM, Hennessy BT, Madiredjo M, Hijmans EM, Beelen K, Linn SC, Gonzalez-Angulo AM, Stemke-Hale K, Hauptmann M, et al. 2007. A functional genetic approach identifies the PI3K pathway as a major determinant of trastuzumab resistance in breast cancer. Cancer Cell 12: 395-402.

Bozic I, Antal T, Ohtsuki H, Carter H, Kim D, Chen S, Karchin R, Kinzler KW, Vogelstein B, Nowak MA. 2010. Accumulation of driver and passenger mutations during tumor progression. Proc Natl Acad Sci 107: 1854518550.

Brooks JD. 2012. Translational genomics: The challenge of developing cancer biomarkers. Genome Res (this issue). doi: 10.1101/gr.124347.111.

Cancer Genome Atlas Research Network. 2008. Comprehensive genomic characterization defines human glioblastoma genes and core pathways. Nature 455: 1061-1068.

Cancer Genome Atlas Research Network. 2011. Integrated genomic analyses of ovarian carcinoma. Nature 474: 609-615.

Carvajal RD, Antonescu CR, Wolchok JD, Chapman PB, Roman RA, Teitcher J, Panageas KS, Busam KJ, Chmielowski B, Lutzky J, et al. 2011. KIT as a therapeutic target in metastatic melanoma. JAMA 305: 2327-2334.

Castellarin M, Warren RL, Freeman JD, Dreolini L, Krzywinski M, Strauss J, Barnes R, Watson P, Allen-Vercoe E, Moore RA, et al. 2012. Fusobacterium nucleatum infection is prevalent in human colorectal carcinoma. Genome Res (this issue). doi: 10.1101/gr.126516.111.

Cheung LW, Hennessy BT, Li J, Yu S, Myers AP, Djordjevic B, Lu Y, StemkeHale K, Zhang F, Ju Z, et al. 2011. High frequency of PIK3R1 and PIK3R2 mutations in endometrial cancer elucidates a novel mechanism for regulation of PTEN protein stability. Cancer Discov 1: 170-185.

Chin L, Hahn WC, Getz G, Meyerson M. 2011. Making sense of cancer genomic data. Genes Dev 25: 534-555.

Ciriello G, Cerami E, Sander C, Schultz N. 2012. Mutual exclusivity analysis identifies oncogenic network modules. Genome Res (this issue). doi: 10.1101/gr.125567.111.

Dewal N, Hu Y, Freedman ML, Laframboise T, Pe'er I. 2012. Calling amplified haplotypes in next generation tumor sequence data. Genome Res (this issue). doi: $10.1101 /$ gr.122564.111.

Druker BJ. 2008. Translation of the Philadelphia chromosome into therapy for CML. Blood 112: 4808-4817.

Gerber DE, Minna JD. 2010. ALK inhibition for non-small cell lung cancer: from discovery to therapy in record time. Cancer Cell 18: 548-551.

Gonzalez-Angulo AM, Ferrer-Lozano J, Stemke-Hale K, Sahin A, Liu S, Barrera JA, Burgues O, Lluch AM, Chen H, Hortobagyi GN, et al. 2011. PI3K pathway mutations and PTEN levels in primary and metastatic breast cancers. Mol Can Ther 10: 1093-1101.

Greenman CD, Pleasance ED, Newman S, Yang F, Fu B, Nik-Zainal S, Jones D, Lau KW, Carter N, Edwards PA, et al. 2012. Estimation of rearrangement phylogeny for cancer genomes. Genome Res (this issue). doi: 10.1101/ gr.118414.110.

Guenthoer J, Diede SJ, Tanaka H, Chai X, Hsu L, Tapscott SJ, Porter PL. 2012. Assessment of palindromes as platforms for DNA amplification in breast cancer. Genome Res (this issue). doi: 10.1101/gr.117226.110.

Harris TJ, McCormick F. 2010. The molecular pathology of cancer. Nat Rev Clin Oncol 7: 251-265.

Hinoue T, Weisenberger DJ, Lange CP, Shen H, Byun HM, Van Den Berg D, Malik S, Pan F, Noushmehr H, van Dijk CM, et al. 2012. Genome-scale analysis of aberrant DNA methylation in colorectal cancer. Genome Res (this issue). doi: 10.1101/gr.117523.110.

Howlader N, Noone AM, Krapcho M, Neyman N, Aminou R, Waldron W, Altekruse SF, Kosary CL, Ruhl J, Tatalovich Z, et al., ed. 2011. SEER Cancer Statistics Review, 1975-2008. National Cancer Institute, Bethesda, MD. http://seer.cancer.gov/csr/1975_2008/ (based on November 2010 SEER data submission, posted to the SEER web site, 2011).

Janku F, Tsimberidou AM, Garrido-Laguna I, Wang X, Luthra R, Hong DS, Naing A, Falchook GS, Moroney JW, Piha-Paul SA, et al. 2011. PIK3CA mutations in patients with advanced cancers treated with PI3K/AKT/ mTOR axis inhibitors. Mol Cancer Ther 10: 558-565. 
Jensen JD, Laenkholm AV, Knoop A, Ewertz M, Bandaru R, Liu W, Hackl W, Barrett JC, Gardner H. 2011. PIK3CA mutations may be discordant between primary and corresponding metastatic disease in breast cancer. Clin Cancer Res 17: 667-677.

Kostic AD, Gevers D, Pedamallu CS, Michaud M, Duke F, Earl AM, Ojesina AI, Jung J, Bass AJ, Tabernero J, et al. 2012. Genomic analysis identifies association of Fusobacterium with colorectal carcinoma. Genome Res (this issue) doi: 10.1101/gr.126573.111.

Liedtke C, Broglio K, Moulder S, Hsu L, Kau SW, Symmans WF, Albarracin C, Meric-Bernstam F, Woodward W, Theriault RL, et al. 2009. Prognostic impact of discordance between triple-receptor measurements in primary and recurrent breast cancer. Ann Oncol 20: 1953-1958.

Liegl-Atzwanger B, Fletcher JA, Fletcher CD. 2010. Gastrointestinal stromal tumors. Virchows Arch 456: 111-127.

Liu P, Cheng H, Santiago S, Raeder M, Zhang F, Isabella A, Yang J, Semaan DJ, Chen C, Fox EA, et al. 2011. Oncogenic PIK3CA-driven mammary tumors frequently recur via PI3K pathway-dependent and PI3K pathway-independent mechanisms. Nat Med 17: 1116-1120.

Lord CJ, Ashworth A. 2008. Targeted therapy for cancer using PARP inhibitors. Curr Opin Pharmacol 8: 363-369.

Macconaill LE, Garraway LA. 2010. Clinical implications of the cancer genome. J Clin Oncol 28: 5219-5228.

Mangala LS, Han HD, Lopez-Berestein G, Sood AK. 2009. Liposomal siRNA for ovarian cancer. Methods Mol Biol 555: 29-42.

Olive KP, Tuveson DA. 2006. The use of targeted mouse models for preclinical testing of novel cancer therapeutics. Clin Cancer Res 12: 5277-5287.

Oltersdorf T, Elmore SW, Shoemaker AR, Armstrong RC, Augeri DJ, Belli BA, Bruncko M, Deckwerth TL, Dinges J, Hajduk PJ, et al. 2005. An inhibitor of Bcl-2 family proteins induces regression of solid tumours. Nature 435: 677-681.

Paez JG, Jänne PA, Lee JC, Tracy S, Greulich H, Gabriel S, Herman P, Kaye FJ, Lindeman N, Boggon TJ, et al. 2004. EGFR mutations in lung cancer: correlation with clinical response to gefitinib therapy. Science 304: 1497-1500

Quintás-Cardama A, Lazar AJ, Woodman SE, Kim K, Ross M, Hwu P. 2008 Complete response of stage IV anal mucosal melanoma expressing KIT Val560Asp to the multikinase inhibitor sorafenib. Nat Clin Pract Oncol 5: 737-740.

Roychowdhury S, Iyer MK, Robinson DR, Lonigro RJ, Wu YM, Cao X, Kalyana-Sundaram S, Sam L, Balbin OA, Quist MJ, et al. 2011. Personalized oncology through integrative high-throughput sequencing: a pilot study. Sci Transl Med 3: 111ra121. doi: 10.1126/ scitranslmed.3003161.

Shaw JA, Page K, Blighe K, Hava N, Guttery D, Ward B, Brown J, Ruangpratheep C, Stebbing J, Payne R, et al. 2012. Genomic analysis of circulating cell-free DNA infers breast cancer dormancy. Genome Res (this issue). doi: 10.1101/gr.123497.111.

Thompson JF, Reifenberger JG, Giladi E, Kerouac K, Gill J, Hansen E, Kahvejian A, Kapranov P, Knope T, Lipson D, et al. 2012. Single-step capture and sequencing of natural DNA for detection of BRCA1 mutations. Genome Res (this issue). doi: 10.1101/ gr.122192.111.

Tsimberidou AM, Iskander NG, Hong DS, Wheler JJ, Fu S, Piha-Paul SA, Naing A, Falchook GS, Janku F, Luthra R, et al. 2011. Personalized medicine in a phase I clinical trials program: The M.D. Anderson Cancer Center Initiative. I Clin Oncol 29: abstract CRA2500.

Turajlic S, Furney SJ, Lambros MB, Mitsopoulos C, Kozarewa I, Geyer FC, MacKay A, Hakas J, Zvelebil M, Lord C, et al. 2012. Whole genome sequencing of matched primary and metastatic acral melanomas. Genome Res (this issue). doi: 10.1101/gr.125591.111.

Vandin F, Upfal E, Raphael BJ. 2012. De novo discovery of mutated driver pathways in cancer. Genome Res (this issue). doi: 10.1101/ gr.120477.111.

Verdine GL, Walensky LD. 2007. The challenge of drugging undruggable targets in cancer: lessons learned from targeting BCL-2 family members. Clin Cancer Res 13: 7264-7270.

Wagle N, Berger MF, Davis MJ, Blumenstiel B, DeFelice M, Pochanard P, Ducar M, Van Hummelen P, MacConaill LE, Hahn WC, et al. 2011. Highthroughput detection of actionable genomic alterations in clinical tumor samples by targeted, massively parallel sequencing. Cancer Discovery doi: 10.1158/2159-8290.

Wang L, Tsutsumi S, Kawaguchi T, Nagasaki K, Tatsuno K, Yamamoto S, Sang F, Sonoda K, Sugawara M, Saiura A, et al. 2012. Whole-exome sequencing of human pancreatic cancers and characterization of genomic instability caused by $M L H 1$ haploinsufficiency and complete deficiency. Genome Res (this issue). doi: 10.1101/ gr. 123109.111

Weidle UH, Maisel D, Eick D. 2011. Synthetic lethality-based targets for discovery of new cancer therapeutics. Cancer Genomics Proteomics 8: $159-171$.

Weinstein IB. 2002. Addiction to oncogenes-the Achilles heal of cancer. Science 297: 63-64.

Xiong Q, Ancona N, Hauser ER, Mukherjee S, Furey TS. 2012. Integrating genetic and gene expression evidence into genome-wide association analysis of gene sets. Genome Res (this issue). doi: 10.1101/ gr.124370.111.

Xu Y, Hu B, Choi AJ, Gopalan B, Lee BH, Kalady MF, Church JM, Ting AH. 2012. Unique DNA methylome profiles in CpG island methylator phenotype colon cancers. Genome Res (this issue). doi: 10.1101/ gr.122788.111. 


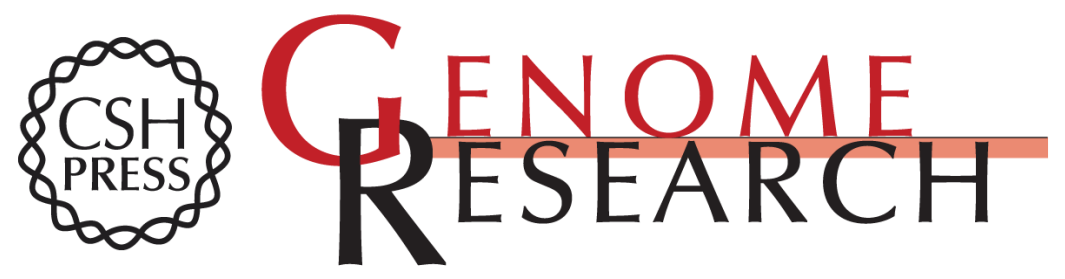

\title{
An emerging toolkit for targeted cancer therapies
}

\author{
Gordon B. Mills
}

Genome Res. 2012 22: 177-182

Access the most recent version at doi:10.1101/gr.136044.111

\section{Related Content Translating genomic information into clinical medicine: Lung cancer as a paradigm \\ Mia A. Levy, Christine M. Lovly and William Pao \\ Genome Res. November, 2012 22: 2101-2108}

References This article cites 26 articles, 11 of which can be accessed free at:

http://genome.cshlp.org/content/22/2/177.full.html\#ref-list-1

Articles cited in:

http://genome.cshlp.org/content/22/2/177.full.html\#related-urls

\section{License}

Email Alerting

Receive free email alerts when new articles cite this article - sign up in the box at the Service top right corner of the article or click here.

\section{Affordable, Accurate Sequencing.}

